\title{
Comparison of Secondary, Backscattered and Low Loss Electron Imaging for Dimensional Measurements in the Scanning Electron Microscope ${ }^{[1,2]}$
}

\author{
Michael T. Postek ${ }^{1}$, John Villarrubia ${ }^{2}$, András E. Vladár ${ }^{3}$ and Atsushi Muto ${ }^{4}$ \\ ${ }^{1-3}$ National Institute of Standards and Technology, Physical Measurement Laboratory, Gaithersburg, MD 20899 \\ ${ }^{4}$ Hitachi High Technologies America, Clarksburg, MD 20871
}

Scanning electron microscopes (SEM) are used for dimensional metrology and process control in many production environments. The accuracy of these SEM measurements has always been important, but is often overshadowed by two other main measurement drivers: throughput and precision. It is slow and often tedious to achieve accuracy and, so it is often ignored in production. Accuracy of a measurement is becoming more of an abiding concern as sub-10 nm semiconductor structures are routinely produced. Hence, the metrology error budget has shrunk, and has become only a couple of atoms, i.e., virtually nonexistent. Clever new measurement and signal collection methods applied to sub-10 nm metrology must be sought for all types of semiconductor nanostructures, nanomaterials and nano-enabled materials to ultimately achieve the needed accurate measurements.

Achieving good SEM measurement accuracy depends on the quality of the acquired image influenced by vibration, drifts, sample contamination and charging, etc., and accounting for specimen-electron beam interactions. New acquisition methods and successful mitigation of detrimental effects can alleviate some of the imaging problems. But, another key element is the application of advanced electron beam-solid state interaction modeling, such as the NIST JMONSEL [3] model to interpret and account for the physics of the signal generation, and help to understand and minimize the various contributions to measurement inaccuracy.

This work is a fundamental comparison of secondary (SE), backscattered (BSE) and low-loss (LLE) electron signals acquired on a new instrument that has high-angle BSE and energy-filtered LLE detectors. Early work indicated that the LLE signal could be advantageous for metrology [4]. When that work was first done, it was very difficult to obtain the needed information because of poor signal-tonoise ratio (SNR) and other instrument-specific geometric limitations. LLE imaging is difficult because LLE represent a small and hence inherently noisier subset of all BSE that have undergone only minimal inelastic interactions with a sample and therefore carry high-resolution, surface-specific information [58]. The use of the conventional backscattered electron signal was shown to be beneficial at low landing energies using a microchannel-plate electron detector [9]. In that work, collection and comparison of the BSE generated images of line structures measured about $10 \%$ narrower, compared to the width of measured SE images [9]. Due to the enhanced emission of low-energy (typically less than $10 \mathrm{eV}$ ) electrons at the sides and corners, there are common circumstances in which the SE intensity increases more abruptly at an edge than the BSE intensity. If width assignments are based on an intensity threshold, SE images would then be interpreted as showing a wider feature than the BSE image. It was anticipated that LLE signal would provide results similar to BSE results. A Hitachi SU 8230 [2] FESEM, equipped with a high-angle and energy-filtered backscattered electron detector, was used to compare the SE, BSE and LLE signals for dimensional measurements of the NIST RM 8820 magnification calibration sample [10]. The design of the new in-lens energy filtered detector improves the LLE signal-to-noise ratio and reduces the geometrical limitations of the early LLE detectors. Work is progressing to apply the NIST JMONSEL model to interpretation of the differences in measurements 
between the modes of electron collection and to ascertain whether better measurement algorithms can be applied to such measurements.

For the first time, point-by point measurement data were able to be obtained simultaneously on a sample using these electron collection modes. Preliminary results (Figure 1) show about a $3 \mathrm{~nm}$ difference between the arbitrary $50 \%$ intensity thresholds of SE and LLE images of the nominally $100 \mathrm{~nm}$ wide $100 \mathrm{~nm}$ tall poly-Si lines on a Si substrate. Therefore, these data are consistent with the results of the earlier work but, in this case, were acquired simultaneously and not serially. Pixel to pixel correlation is now possible. Modeling, to verify and understand this difference further, is currently in progress. Clearly, this points to serious measurement issues encountered by blindly applying measurement algorithms without considering the underlying physics provided by applying model-based metrology.

The potential value of BSE and LLE has not been fully exploited for dimensional metrology, but has not been forgotten. Some of the early results and further experimental and modeling work coupled with modeling are sufficiently promising that prompt continued exploration into the possibilities that LLE affords to metrology in standards development and to determine the necessary information related to design parameters necessary for its implementation.
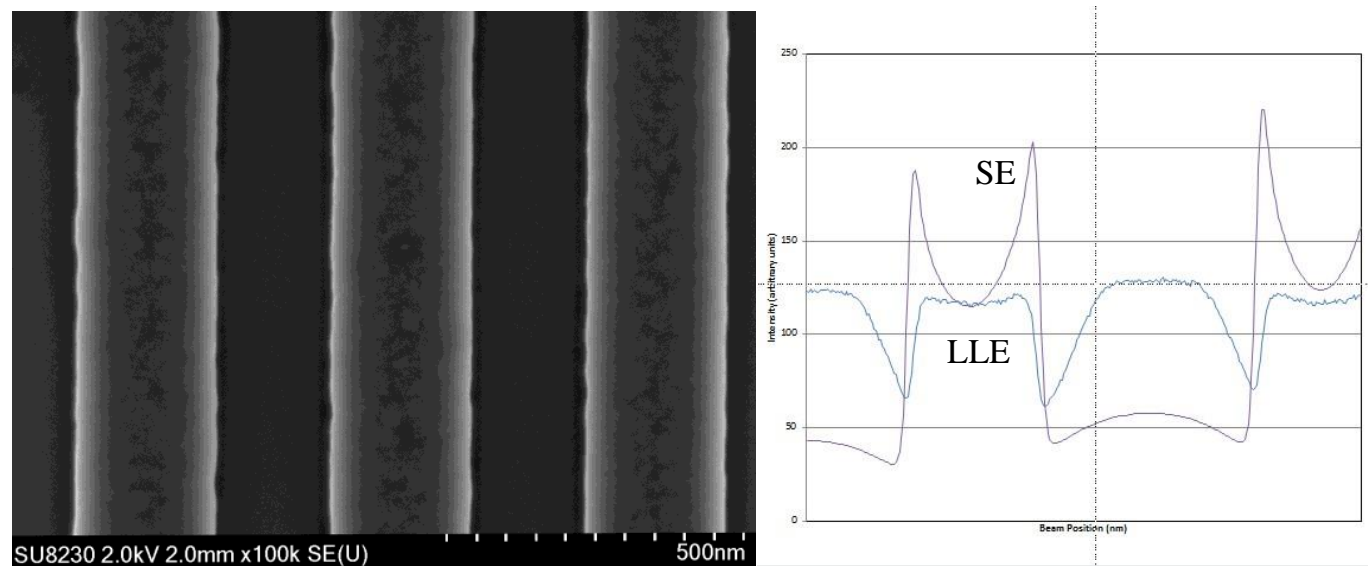

Figure 1. (Left) SE image of RM 8820, the nominal pitch of the lines shown is $425 \mathrm{~nm}$. (Right) SE and LLE linescans from Reference Material 8820 images simultaneously recorded at $2 \mathrm{kV}$. The nominal pitch of the lines measured is $200 \mathrm{~nm}$.

\section{References:}

[1]Contribution of the National Institute of Standards and Technology; not subject to copyright.

[2]Certain commercial equipment is identified in this report to adequately describe the experimental procedure. Such identification does not imply recommendation or endorsement by the National Institute of Standards and Technology, nor does it imply that the equipment identified is necessarily the best available for the purpose.

[3] J.S. Villarrubia et al., Proc. SPIE 6518 (2007) p. 65180K.

[4]M. Postek et al., SCANNING 23(5), (2001), p. 298.

[5]O. C. Wells, Appl Phys Lett 16(4), (1970), p.151.

[6]O. C. Wells, Appl Phys Lett 19(7), (1971), p. 232.

[7]O. C. Wells, Scan Electron Microsc, 1, IITRI Chicago, (1972), p. 43.

[8]O. C. Wells, Appl. Phys. Lett. 49(13), (1986) p. 764.

[9]M. Postek et al., Rev Sci. Instrum, 61(12), (1990), p. 3750.

[10]https://www-s.nist.gov/srmors/view_detail.cfm?srm=8820 